\title{
Submuköse Raumforderung: Eine diagnostische Herausforderung
}

\begin{abstract}
Gut- oder bösartig? Das ist die Gretchenfrage, wenn bei der Gastroskopie zufällig ein submuköser Tumor diagnostiziert wird. Wann sollte man die feingewebliche Diagnose erzwingen und wann ist zunächst nur eine Verlaufskontrolle angezeigt?
\end{abstract}

„Von einer submukösen Raumforderung spricht man, wenn eine Raumforderung mit normal wirkender Schleimhaut vorliegt", erläuterte Professor Alexander Arlt, Kiel. Ein solcher Befund sei sehr selten, er finde sich nur bei $0,36 \%$ aller Routine-Ösophago-Gastro-Duodenoskopien. In der Regel handele es sich um einen asymptomatischen Zufallsbefund. Das differenzialdiagnostische Spektrum ist weit. Es reicht von benignen Tumoren wie Lipomen und Zysten bis hin zu potenziell malignen Tumoren. Dazu gehören gastrointestinale Stromatumoren (GIST), Lymphome, neuroendokrine Tumoren, Metastasen und Glomustumoren. „Viele Patienten werden durch die Mitteilung eines solchen Befundes stark verunsichert", betonte Arlt. Oft werde dann eine ungeordnete Diagnostik betrieben, die auch nicht selten zu einer Übertherapie führe.

\section{Immer zunächst endoskopischer Ultraschall}

Diagnose erzwingen oder Verlaufskontrolle? Dies sei, so Arlt, die entscheidende Frage. Schon bei der Endoskopie könnten wichtige Informationen gewonnen werden. So spricht eine weiche Konsistenz für eine Zyste, ein Gefäß oder ein Lymphom. Doch für die weitere Beurteilung ist der endoskopische Ultraschall unverzichtbar. Mit ihm gelingt zunächst die exakte Größenbestimmung. Entscheidend aber ist zunächst, dass nur mittels endoskopischem Ultraschall echte intramurale Tumoren von Pseudotumoren abgegrenzt werden können. „Die Kompression von außen durch den linken Leberlappen, Milz oder Lymphknoten kann einen submukösen Tumor vortäuschen", so Arlt. Bei echten Tumoren ist dann die Echogenität entscheidend für das weitere Vorgehen. Bei echoreichen Befunden handelt es sich um ein Lipom und bei einem echofreien Tumor dürften ein Lymphangiom oder eine Zyste oder Varizen vorliegen. „All diese Befunde sind immer benigne und müssen deshalb auch nicht kontrolliert oder weiter abgeklärt werden“, erklärte Arlt.

\section{Echoarme Tumoren sind das Problem}

Ein diagnostisches Problem sind die echoarmen submukösen Tumoren. Fachgesellschaften empfehlen eine weitere Abklärung erst ab einer Größe von $2 \mathrm{~cm}$. „Im praktischen Alltag wird auch aus psychologischen Gründen, nämlich weil der Patient oft verunsichert ist, auch bei kleineren Befunden meist eine weitere Diagnostik durchgeführt“, so Arlt. Eine eindeutige Dignitätsbeurteilung sei mithilfe des Ultraschalls auch in Kombination mit dem B-Bild nur in zirka $50 \%$ der Fälle möglich. Bei einer Genauigkeit von 50 \% könnte man auch eine Münze werfen. Um einen potenziell malignen Tumor zweifelsfrei zu erkennen, sollte eine Histologie angestrebt werden.

\section{Der Weg zur Probengewinnung}

Früher wurde in der Regel eine Schlüssellochbiopsie durchgeführt. Doch eine solche ist nicht ganz ungefährlich, in zirka $3 \%$ kommt es zu einer stärkeren Blutung. Auch ist die Sensitivität im Vergleich zur heute üblichen endoskopischen Mukosaresektion eher niedrig. „Eine solche endoskopische Ultraschall-gesteuerte Entdeckelung ist sensitiver und auch nicht gefährlicher", erläuterte Arlt. Wenn im endoskopischen Ultraschall unterhalb des submukösen Tumors die Muscularis erhalten ist, ist sogar eine En-bloc-Resektion mittels endoskopischer Submukosadissektion möglich. „Dieses Verfahren bietet dann Diagnosesicherheit und Therapie in einem", so Arlt. Dies gelinge in $94 \%$ der Fälle. Ist die Muscularis nicht sicher erhalten, so sinkt die Resektionsrate auf $60 \%$ und das Perforationsrisiko steigt an. Dann sollte eventuell im Einzelfall auch eine operative Resektion diskutiert werden, wobei man aber die Invasivität und Morbidität des Eingriffs bedenken sollte. „Dies kann eine Übertherapie darstellen, die mit einer erhöhten Morbidität assoziiert sein kann", mahnte Arlt. Eine definitive Diagnose anzustreben sei nur vertretbar, wenn die submuköse Raumforderung größer als $2 \mathrm{~cm}$ sei und sonografisch ein echoarmer oder inhomogener Befund oder eine unscharfe Begrenzung zur Darstellung komme oder der Tumor schnell wachse.

Dr. med. Peter Stiefelhagen

Multimodale Therapie von GIST: Zufallsbefund submuköse Raumforderung im GI-Trakt. Viszeralmedizin 2017, 14.9.2107 in Dresden

\section{Risiko Anastomosenkarzinom nach Gastrektomie}

Ein Anastomosenkarzinom ist selten. In großen Serien mit 300 bis $1.000 \mathrm{~Pa}$ tienten werden $2 \%$ bis $4 \%$ Karzinome entdeckt, erläuterte Professor Markus Menges, Diakonie-Klinikum Schwäbisch Hall. Mehr als 90\% der Rezidive entwickeln sich in den ersten drei Jahren nach dem operativen Eingriff. Nach distaler Gastrektomie sind auch Spätrezidive möglich. Wird wegen eines Karzinoms reseziert, kann das Rezidiv im Magenstumpf nach zwei bis 22 Jahren auftreten (im Mittel: zwölf Jahre), nach Resektion wegen benigner Ursache sogar noch nach 51 Jahren, im Mittel nach 30 Jahren. Die Operationstechnik scheint für das Risiko relevant: Karzinome im Magenstumpf treten nach B-I-Resektionen früher auf als nach B-II-Resektionen (zwölf vs. 32 Jahre). Wird allerdings wegen benigner Ursache reseziert, sind Karzino- me nach B-II-Resektion häufiger. Diskutiert wird über Strategien, um ein Rezidiv frühzeitig zu erkennen. Bei partieller Magenresektion aus benigner Ursache sind regelmäßige endoskopische Kontrollen laut Menges normalerweise nicht indiziert. Als eventuelle Ausnahme nannte er den „klassischen B-II-Magen“. Bei distaler Magenresektion wegen eines Malignoms können aus seiner Sicht regelmäßige endoskopische Kontrollen sinnvoll sein. Das Intervall wäre dann insbesondere abhängig vom Vorliegen einer intestinalen Metaplasie oder einer chronischen B-Gastritis.

Dr. Beate Fessler

Folgekrankheiten nach chirurgischen Resektionen: Anastomosenkarzinom nach Magenresektion. Viszeralmedizin 2017, 14.9.2107 in Dresden 